\title{
Evaluation of Marine Emergency Rescue Ability Based on Fuzzy Network Analysis
}

\author{
XuanCheng Zhang ${ }^{1, \text { a }}$, Chunhui Zhou ${ }^{2, b}$, Jingqun Huo ${ }^{3, c}$, Shirui Ren , Jirong \\ Jiang , Zhiqi Li and Yinben Zhang \\ ${ }^{1}$ School of Navigation, Wuhan University Of Technology, China \\ ${ }^{2}$ School of Navigation, Wuhan University Of Technology, China \\ a359639762@qq.com, bchunhui@whut.edu.cn, c hjq0121512370605@163.com
}

\begin{abstract}
Key words:Interpretation Structure Model Method; Network Analysis Method; Fuzzy Comprehensive Evaluation; Evaluation of Maritime Rescue Capability

Abstract:The evaluation of the rescue capability of the maritime department plays an important role in improving the emergency rescue capability of maritime emergencies and optimizing the emergency management of emergencies, which can reduce the occurrence of similar emergencies at sea. But the emergency rescue operations at sea are complex and random, which leads to the complexity of the assessment process of emergency rescue capability at sea. In this paper, we use the explanatory structural model method (ISM) to analyze the relationship between the evaluation indexes of maritime rescue ability, and then use the network analysis method to determine the weight of each index. Considering the feedback relationship between indicators, the weight of the index is determined reasonably and the model Scientific and reliability, combined with fuzzy comprehensive evaluation method to evaluate the ability to rescue. Maritime departments can be based on the evaluation results combined with the weight of indicators, targeted to improve, and thus effectively improve the maritime emergency rescue capabilities.
\end{abstract}

\section{Introduction}

With the vigorous development of the international shipping industry, maritime transport in the development of economic globalization occupy an increasingly important position. The frequent occurrence of maritime accidents has also brought a lot of casualties and property damage, but also has a huge adverse impact on the navigation environment, navigation order, the marine environment and the public interest of society. In order to improve the emergency rescue capability at sea, it is very important to evaluate the emergency rescue capability of the sea and to point out the existing problems and put forward the corresponding solutions. If the appropriate risk control system can be established according to the existing problems and the corresponding solutions, it will be able to improve the maritime emergency rescue capabilities effectively, reduce the loss caused by maritime accidents, but also to avoid the sea emergency rescue capacity to enhance the process of waste of resources.

In the emergency rescue capability evaluation on the disaster, domestic and foreign scholars have also carried out a lot of related research. In the study of coal mine emergency rescue capability, Yang Li and others used improved analytic hierarchy process, entropy weight method and fuzzy comprehensive evaluation method to establish the coal mine emergency rescue capability evaluation model, as the basis for the construction of the coal mine emergency rescue 
capability index evaluation system. Duan Zongtao et al in the establishment of expressway emergency rescue capability evaluation index system used AHP and fuzzy comprehensive evaluation method to design the evaluation results calculation model, in which the comprehensive membership degree was transformed into a comprehensive evaluation process to simplify the evaluation process. Miao Chenglin et al divided the emergency into three steps which is pre-emergencies, things and after to carry out the research of prevention, detection, control and recovery, analyzed the relationship between the evaluation indicators of coal mine enterprise emergency rescue ability, put forward the theoretical assumptions of the relationship between the evaluation indicators. And through empirical analysis, they used structural equation model to verify the hypothesis, proposed to improve the coal mine enterprise emergency rescue ability proposal. Daniel da Silva avanzi et al. used multi - criteria decision analysis (MCDA) to establish a support assessment system for disaster management (DM) to assess potential public entities and locations for collaboration.

According to these existing research results it is observed that for the disaster in the emergency rescue capability evaluation has been a lot, but for the assessment of the emergency rescue capability at sea is very little. There is no mature solution to solve this problem effectively. Different from the traditional evaluation model using the AHP method to determine the index weight, this paper uses the Analytic Network Process (ANP), which considers the relationship between indicators to sort the importance of the index of emergency rescue capability in the sea. Then, the fuzzy comprehensive evaluation method is used to evaluate the emergency rescue capability of the rescue department. The evaluation results can truly reflect the excellent degree of emergency rescue capability of the rescue department. According to the importance ranking of the rescue ability evaluation index, the weakness or lack of rescue ability can be found and provide accurate and effective reference for further improving the emergency rescue capability at sea.

\section{Evaluation Model of Maritime Rescue Ability}

\section{Rescue Ability Evaluation Index System}

The evaluation system of maritime rescue capability includes the interrelationship between indicators and indicators.

\section{Maritime Rescue Capability Indicators}

Through the analysis of existing research results and the actual rescue case, we summed up the upper and lower levels of offshore rescue capacity evaluation indicators shown in Figure 1. The upper level indicators directly determine the comprehensive ability to rescue, the lower indicators through the impact of upper indicators indirectly determine the comprehensive ability to rescue. 


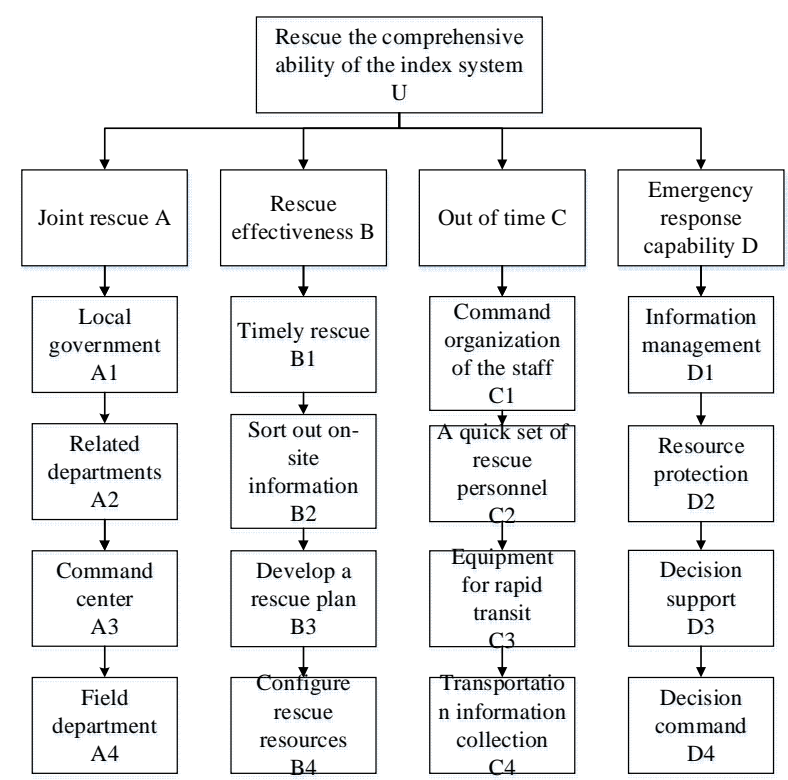

\begin{tabular}{|c|c|c|c|c|c|c|c|c|}
\hline the layers and index values & $\mathrm{A}$ & 0.2109 & $\mathrm{~B}$ & 0.3388 & $\mathrm{C}$ & 0.3388 & $\mathrm{D}$ & 0.0647 \\
\hline \multirow{3}{*}{$\begin{array}{c}\text { Target level indicators and } \\
\text { Corresponding final index } \\
\text { values }\end{array}$} & $\mathrm{A} 1$ & 0.0046 & $\mathrm{~B} 1$ & 0.0801 & $\mathrm{C} 1$ & 0.0519 & $\mathrm{D} 1$ & 0.0278 \\
\cline { 2 - 10 } & $\mathrm{A} 2$ & 0.0801 & $\mathrm{~B} 2$ & 0.0076 & $\mathrm{C} 2$ & 0.0850 & $\mathrm{D} 2$ & 0.0579 \\
\cline { 2 - 10 } & $\mathrm{A} 3$ & 0.0519 & $\mathrm{~B} 3$ & 0.0850 & $\mathrm{C} 3$ & 0.1466 & $\mathrm{D} 3$ & 0.0159 \\
\cline { 2 - 10 } & $\mathrm{A} 4$ & 0.0278 & $\mathrm{~B} 4$ & 0.0579 & $\mathrm{C} 4$ & 0.0159 & $\mathrm{D} 4$ & 0.1411 \\
\hline
\end{tabular}

Fig.1Assessment indicators of marine rescue abilities

\section{The Determination of the Relationship Between the Evaluation Indexes}

After the evaluation index is determined, it is necessary to determine the relationship between the indicators. Interpretative Structural Model (ISM) is a structural modeling technique proposed by J Warfield. The model can be used to determine the interrelationships of the evaluation capability of offshore rescue capability, specific steps are as follows:

(1) Create the adjacency matrix

The relationship between the evaluation indexes of the emergency response capability is described by the adjacent matrix $\mathrm{A}$. The element $\mathrm{a}_{\mathrm{ij}}$ in $\mathrm{A}$ is used to represent the influence relation between the influencing factors, and the above 16 indexes are arranged according to S0-S15, as shown in (1).

$$
a_{i j}= \begin{cases}1 & \left(S_{i} \text { and } S_{j} \text { directly before the first }\right) \\ 0 & \left(S_{i} \text { and } S_{j} \text { are not directly related }\right)\end{cases}
$$

After the study group discussed and consulted the experts, we obtained the offshore rescue capability index adjacency matrix A as shown in Table 1. 


\begin{tabular}{|c|c|c|c|c|c|c|c|c|c|c|c|c|c|c|c|c|}
\hline & $\mathrm{S}_{0}$ & $\mathrm{~S}_{1}$ & $\mathrm{~S}_{2}$ & $\mathrm{~S}_{3}$ & $\mathrm{~S}_{4}$ & $\mathrm{~S}_{5}$ & $\mathrm{~S}_{6}$ & $\mathrm{~S}_{7}$ & $\mathrm{~S}_{8}$ & $\mathrm{~S}_{9}$ & $\mathrm{~S}_{10}$ & $\mathrm{~S}_{11}$ & $\mathrm{~S}_{12}$ & $\mathrm{~S}_{13}$ & $\mathrm{~S}_{14}$ & $\mathrm{~S}_{15}$ \\
\hline $\mathrm{S}_{0}$ & 1 & 0 & 0 & 0 & 0 & 0 & 0 & 0 & 0 & 0 & 0 & 0 & 0 & 0 & 0 & 0 \\
\hline $\mathrm{S}_{1}$ & 1 & 1 & 0 & 0 & 0 & 0 & 0 & 0 & 0 & 0 & 0 & 0 & 0 & 0 & 0 & 0 \\
\hline $\mathrm{S}_{2}$ & 1 & 1 & 1 & 1 & 1 & 1 & 1 & 0 & 0 & 0 & 0 & 1 & 0 & 0 & 0 & 0 \\
\hline $\mathrm{S}_{3}$ & 1 & 1 & 0 & 1 & 1 & 1 & 1 & 0 & 0 & 0 & 0 & 0 & 0 & 0 & 0 & 0 \\
\hline $\mathrm{S}_{4}$ & 1 & 1 & 0 & 0 & 1 & 0 & 0 & 1 & 0 & 0 & 0 & 0 & 0 & 0 & 0 & 0 \\
\hline $\mathrm{S}_{5}$ & 1 & 1 & 0 & 0 & 0 & 1 & 0 & 1 & 1 & 0 & 0 & 0 & 0 & 0 & 0 & 0 \\
\hline $\mathrm{S}_{6}$ & 1 & 1 & 0 & 0 & 0 & 0 & 1 & 1 & 1 & 0 & 0 & 0 & 0 & 0 & 0 & 0 \\
\hline $\mathrm{S}_{7}$ & 1 & 0 & 0 & 0 & 0 & 0 & 0 & 1 & 0 & 0 & 0 & 0 & 0 & 0 & 0 & 0 \\
\hline $\mathrm{S}_{8}$ & 1 & 1 & 0 & 0 & 0 & 0 & 0 & 1 & 1 & 0 & 0 & 0 & 1 & 0 & 0 & 0 \\
\hline $\mathrm{S}_{9}$ & 1 & 1 & 0 & 0 & 0 & 0 & 0 & 1 & 0 & 1 & 0 & 0 & 1 & 0 & 0 & 0 \\
\hline $\mathrm{S}_{10}$ & 1 & 1 & 0 & 0 & 0 & 0 & 0 & 1 & 0 & 0 & 1 & 0 & 1 & 0 & 0 & 0 \\
\hline $\mathrm{S}_{11}$ & 1 & 0 & 0 & 0 & 0 & 0 & 0 & 1 & 0 & 0 & 0 & 1 & 1 & 0 & 0 & 0 \\
\hline $\mathrm{S}_{12}$ & 1 & 0 & 0 & 0 & 0 & 0 & 0 & 0 & 0 & 0 & 0 & 0 & 1 & 0 & 0 & 0 \\
\hline $\mathrm{S}_{13}$ & 1 & 1 & 0 & 0 & 0 & 0 & 0 & 0 & 0 & 0 & 0 & 0 & 1 & 1 & 0 & 0 \\
\hline $\mathrm{S}_{14}$ & 1 & 0 & 0 & 0 & 0 & 0 & 0 & 0 & 0 & 0 & 0 & 0 & 1 & 0 & 1 & 1 \\
\hline $\mathrm{S}_{15}$ & 1 & 0 & 0 & 0 & 0 & 0 & 0 & 0 & 0 & 0 & 0 & 0 & 1 & 0 & 0 & 1 \\
\hline
\end{tabular}

Table 1 Adjacency matrix A

\section{(2) Create the reachable matrix}

The degree that each node of a directed graph can reach through certain path is described by reachability matrix $\mathrm{M}$. Boolean matrix algorithm is used to compute reachability matrix. Because adjacency matrix A belongs to Boolean matrix.

$$
M=(A+I)^{k}=(A+I)^{(k-1)} \neq(A+I)^{(k-2)} \neq \mathrm{L}(A+I) \quad(k \leq n-1)
$$

The reachable matrix $\mathrm{M}$ is used to represent all direct and indirect relationships between the various rescue capability indicators.

(3) Build hierarchical structure chart

In reachability matrix $\mathrm{M}$, the reachability set $\mathrm{R}(\mathrm{Si})$ is established. It Represents a collection of all indexes that can be reached from the index Si. And it sums up the direct impact of each index. The antecedent set $\mathrm{A}(\mathrm{Si})$ represents all the index sets that can reach the target $\mathrm{Si}$. Make $C\left(S_{i}\right)=R\left(S_{i}\right) \cap A\left(S_{i}\right)$ indicate the interaction with indicator $\mathrm{Si}$, and the $\mathrm{R}\left(\mathrm{S}_{\mathrm{i}}\right) 、 \mathrm{~A}\left(\mathrm{~S}_{\mathrm{i}}\right)$ 和C $\left(\mathrm{S}_{\mathrm{i}}\right)$ of this reachability matrices are shown in Table 2. 


\begin{tabular}{|c|c|c|c|}
\hline $\mathrm{S}_{\mathrm{i}}$ & $\mathrm{R}\left(\mathrm{S}_{\mathrm{i}}\right)$ & A $\left(S_{i}\right)$ & $\mathrm{C}\left(\mathrm{S}_{\mathrm{i}}\right)$ \\
\hline 0 & 0 & all & 0 \\
\hline \multirow[t]{2}{*}{1} & 0,1 & $1,2,3,4,5,6,8,9,10,13$ & 1 \\
\hline & & 14,15 & \\
\hline \multirow[t]{2}{*}{2} & $0,1,2,3,4,5,6$ & 2 & 2 \\
\hline & $7,8,11,12$ & & \\
\hline \multirow[t]{2}{*}{3} & $0,1,3,4,5,6,7$ & 2,3 & 3 \\
\hline & 8,12, & & \\
\hline 4 & $0,1,4,7$ & $2,3,4$ & 4 \\
\hline 5 & $0,1,5,7,8,12$ & $2,3,5$ & 5 \\
\hline 6 & $0,1,6,7,8,12$ & $2,3,6$ & 6 \\
\hline 7 & 0,7 & $2,3,4,5,6,7,8,9,10,11$ & 7 \\
\hline 8 & $0,1,7,8,12$ & $2,3,5,6,8$ & 8 \\
\hline 9 & $0,1,7,9,12$ & 9 & 9 \\
\hline 10 & $0,1,7,10,12$ & 10 & 10 \\
\hline 11 & $0,7,11,12$ & 2,11 & 11 \\
\hline \multirow[t]{2}{*}{12} & 0,12 & $2,3,5,6,8,9,10,11,12,1$ & 12 \\
\hline & & $3,14,15$ & \\
\hline 13 & $0,1,12,13$ & 13 & 13 \\
\hline 14 & $0,1,12,14,15$ & 14 & 14 \\
\hline 15 & $0,1,12,15$ & 14,15 & 15 \\
\hline
\end{tabular}

Table2 $\mathrm{R}(\mathrm{Si}) 、 \mathrm{~A}(\mathrm{Si})$ and $\mathrm{C}(\mathrm{Si})$ of reachable matrix $\mathrm{M}$

Once you have found the highest-level feature set, you can draw the corresponding rows and columns from the reachable matrix, and then continue looking for the new highest-level elements from the remaining reachable matrices. And so on, you can find out the highest set of elements at all levels ${ }^{[6]}$. Solutions:

$$
\begin{gathered}
\mathrm{L} 1=\left\{\mathrm{S}_{0}\right\} \quad(3) \quad \mathrm{L} 2=\left\{\mathrm{S}_{1}, \mathrm{~S}_{7}, \mathrm{~S}_{12}\right\} \quad(4) \quad \mathrm{L} 3=\left\{\mathrm{S}_{4}, \mathrm{~S}_{8}, \mathrm{~S}_{9}, \mathrm{~S}_{10}, \mathrm{~S}_{11}, \mathrm{~S}_{13}\right\} \quad(5) \\
\mathrm{L} 4=\left\{\mathrm{S}_{5}, \mathrm{~S}_{6}, \mathrm{~S}_{15}\right\} \quad(6) \quad \mathrm{L} 5=\left\{\mathrm{S}_{3}, \mathrm{~S}_{14}\right\} \quad(7) \quad \mathrm{L} 6=\left\{\mathrm{S}_{2}\right\} \quad(8)
\end{gathered}
$$

According to the classification of $\mathrm{L}_{\mathrm{i}}$, the relationship between the superior index and the subordinate index is established by combining the reachable matrix, and the explanatory structure model of the sea emergency response evaluation index is shown in Fig. 1. 


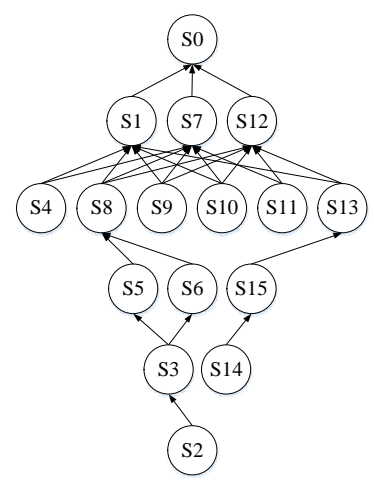

Fig.2 Interpretive structural model of marine emergency response abilities assessment indicators

\section{Evaluation of the importance of evaluation index of rescue ability}

Identifying and focusing on strengthening the construction of relatively important indicators can help the maritime sector to improve the maritime emergency rescue capability effectively. In the past studies, the AHP method was used to sort the evaluation indexes of the rescue capability of the sea. However, the AHP method independently analyzed the elements of each layer, and only emphasized that the upper elements play a dominant role in the lower elements. And the sea rescue work is a complex system, there is an internal feedback between the indicators in its corresponding emergency response capability assessment of the sea. Therefore, it is not accurate to use the traditional AHP method to solve the complex sea emergency rescue capability evaluation problem.

Therefore, we use the Analytic Network Process (ANP), which considers the interrelationships, to sort the importance of emergency rescue capability indicators.

ANP is a system decision-making method by the United States Pittsburgh University professor Satty on the basis of AHP. ANP divides the complex system with internal relations between the indicators into the network layer composed of the control layer composed of the problem, the target and the decision quasi-layer and the dominated index. The indicators in the decision-making quasi-independent are independent of each other, and the indicators in the network layer have feedback.

\section{Determination of network structure for evaluation of maritime rescue capability}

The feedback relationship between the evaluation index of the maritime rescue ability is mainly based on the following criteria: "Commentary Excellent - Comment Excellent / Poor" - The evaluation index of the previous index affects the evaluation grade of the latter index, "Commentary - Comment" - The previous index and the latter indicator has nothing to do. Then we synthesize practical experience and expert's advice, and determine the sea rescue capacity evaluation network structure diagram shown in Figure 3.

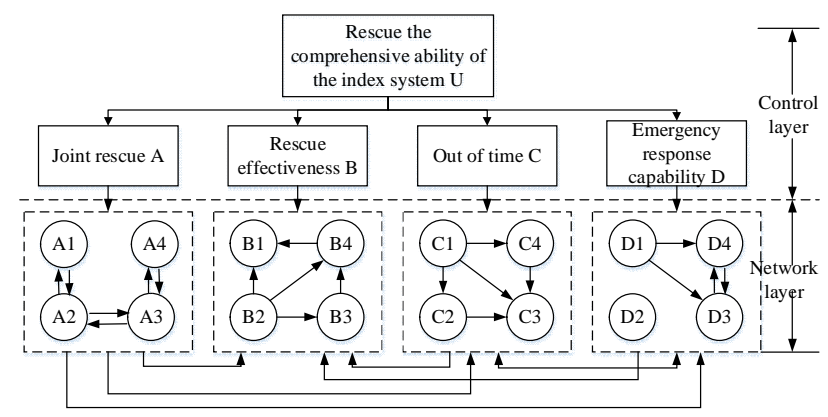

Fig.3 Sea rescue capacity evaluation network structure diagram 


\section{Ranking the importance of the four criteria layer indicators}

Since the criterion layer indicators are independent of each other, the corresponding weights can be determined by the ANP method. The index is determined according to the scale of $1 \sim 9$, and the relative importance degree of the index is compared with the Delphi method, Which is constructed as a pair of comparison matrix B. Then we calculate the eigenvector by matrix B and normalize it to obtain the weight vector of each criterion layer. Table 3 is the scale of 1 to 9 , where $S_{\mathrm{ij}}$ is the importance of indicator $S_{\mathrm{i}}$ relative to $S_{\mathrm{j}}$, Table 4 is the pairwise comparison matrix $\mathrm{B}$ and the weight vector of the criterion layer index.

\begin{tabular}{|c|c|}
\hline$S_{i, j}$ & illustration \\
\hline 1 & $\begin{array}{l}\text { Indicators of } S_{i} \text { and } S_{j} \text { are equally } \\
\text { important }\end{array}$ \\
\hline 3 & $\mathrm{~S}_{\mathrm{i}}$ is a little more important than $\mathrm{S}_{\mathrm{j}}$ \\
\hline 5 & $\mathrm{~S}_{\mathrm{i}}$ is more important than $\mathrm{S}_{\mathrm{j}}$ \\
\hline 7 & $S_{i}$ is more important than $S_{j}$ \\
\hline 9 & $S_{\mathrm{i}}$ is more important than $S_{j}$ absolutely \\
\hline
\end{tabular}

(Note: $2,4,6,8$, respectively, said the two adjacent standard value to determine the median)

Table $31 \sim 9$ scale table

\begin{tabular}{cccccc}
\hline Criterion layer & A & B & C & D & Weight value w1 \\
\hline A & 1 & $1 / 2$ & 1 & 3 & 0.2109 \\
B & 2 & 1 & 1 & 5 & 0.3388 \\
C & 2 & 1 & 1 & 5 & 0.3388 \\
D & $1 / 3$ & $1 / 5$ & $1 / 5$ & 1 & 0.0647 \\
\hline
\end{tabular}

Table 4 Pairwise comparison matrix B and the weight vector of criterion layer

Consistency test of the results, random consistency ratio $\mathrm{CR}=0.08643<0.1$, through the consistency test.

\section{Order of importance of network layer index}

There are multiple criteria for the evaluation of the network structure of the sea emergency rescue. Ranking the importance of the network layer indicators, we first need to determine the unweighted hyper-matrix of the subordinate indicators of each criterion. $\circ$ Taking the joint rescue criterion as an example, the paper analyzes the judgment matrix with the Delphi method, and then uses the characteristic root method to determine the weight vector. Specific steps include the following steps:

(1) Determine the unweighted super-matrix W

According to figure 2, it takes the local government A1 as the criterion. According to comparing the relative importance of the guidelines A1 with the relevant departments A2, command center A3 and site department A4. The sorting vector $\left(\omega_{11}^{(11)}, \omega_{12}^{(11)}, \omega_{13}^{(11)}\right)^{T}$ is obtained by combining the characteristic root method. Then the guidelines are given by the relevant departments A2, command center A3 and site department A4. Repeat the above steps, find the corresponding sort vector, and integrate the vector matrix $W_{11}$ :

$$
W_{11}=\left[\begin{array}{llll}
\omega_{11}^{(11)} & \omega_{11}^{(12)} & \omega_{11}^{(13)} & \omega_{11}^{(14)} \\
\omega_{12}^{(11)} & \omega_{12}^{(12)} & \omega_{12}^{(13)} & \omega_{12}^{(14)} \\
\omega_{13}^{(11)} & \omega_{13}^{(11)} & \omega_{13}^{(13)} & \omega_{13}^{(14)} \\
\omega_{14}^{(11)} & \omega_{14}^{(12)} & \omega_{14}^{(13)} & \omega_{14}^{(14)}
\end{array}\right]=\left[\begin{array}{cccc}
1 & \frac{1}{3} & \frac{1}{5} & \frac{1}{5} \\
3 & 1 & \frac{1}{3} & \frac{1}{5} \\
5 & 3 & 1 & 1 \\
5 & 3 & 1 & 1
\end{array}\right]
$$


Similarly, the vector matrix of the other elements of the criterion layer is obtained and integrated. The unweighted matrix are established. W:

$$
W=\left[\begin{array}{llll}
W_{11} & W_{12} & W_{12} & W_{14} \\
W_{21} & W_{22} & W_{23} & W_{24} \\
W_{31} & W_{32} & W_{33} & W_{34} \\
W_{41} & W_{42} & W_{43} & W_{44}
\end{array}\right]=\left[\begin{array}{llll}
0.0679 & 0.1524 & 0.3899 & 0.3899 \\
0.5261 & 0.0872 & 0.2042 & 0.1826 \\
0.1322 & 0.4165 & 0.3829 & 0.0673 \\
0.0712 & 0.3174 & 0.2358 & 0.3756
\end{array}\right]
$$

(2) Build the matrix of weights a

Since the size of the influence of each network layer index is judged by comparing the corresponding elements, the column vectors of the super-matrix $\mathrm{W}$ composed of each vector matrix are not normalized. In order to solve the above problems, the group of elements is compared, the judgment matrix is obtained, and the eigenvector is calculated to obtain the weight matrix a:

$$
a=W^{T}=\left[\begin{array}{llll}
a_{11} & a_{12} & a_{13} & a_{14} \\
a_{21} & a_{22} & a_{23} & a_{24} \\
a_{31} & a_{32} & a_{33} & a_{34} \\
a_{41} & a_{42} & a_{43} & a_{44}
\end{array}\right]
$$

In the formula: $a_{i j}=0$ indicates that the index $\mathrm{i}$ does not affect the index $\mathrm{j}$, and the sum of the columns of the matrix a is 1 .

(3) Constructing weighted super matrices $\bar{W}$

The weighting matrix a is multiplied by the unweighted super matrix $\mathrm{W}$ to find the weighted super matrix $\bar{W}$. Each column vector element of $\bar{W}$ represents the size of the corresponding network layer index affecting the index on this column. Take zero if there is no effect.

$$
\begin{aligned}
\bar{W} & =a \cdot W \\
& =W^{T} \cdot W \\
& =\left[\begin{array}{llll}
a_{11} W_{11} & a_{12} W_{12} & a_{13} W_{13} & a_{14} W_{14} \\
a_{21} W_{21} & a_{22} W_{22} & a_{23} W_{23} & a_{24} W_{24} \\
a_{31} W_{31} & a_{32} W_{32} & a_{33} W_{33} & a_{34} W_{34} \\
a_{41} W_{41} & a_{42} W_{42} & a_{43} W_{43} & a_{44} W_{44}
\end{array}\right]
\end{aligned}
$$

(4) Weighted super matrix $\bar{W}$ takes the limit

Due to the relationship between feedback and negative feedback in indicators of the offshore rescue capacity evaluation network, the two indicators can be compared by direct and indirect means, thus increasing the complexity of determining the priority of the indicators. Therefore, in order to determine the priority of the stable index, it is necessary to obtain the limit $\bar{W}_{l}$ of the weighted super matrix $\bar{W}$ :

$$
\begin{aligned}
\bar{W}_{l} & =\lim _{t \rightarrow \infty} \bar{W}^{t} \\
& =\left[\begin{array}{llll}
0.0046 & 0.0801 & 0.0519 & 0.0278 \\
0.0801 & 0.0076 & 0.0850 & 0.0579 \\
0.0519 & 0.0850 & 0.1466 & 0.0159 \\
0.0278 & 0.0579 & 0.0159 & 0.1411
\end{array}\right]
\end{aligned}
$$

The column $\mathbf{J}$ of $\bar{W}_{l}$ is the limit relative sorting vector of the relative index $\mathbf{J}$ in the network 
layer under the corresponding criterion. That is the first row represents the weight corresponding w2 to the network layer under the standard A. The last line of $\bar{W}_{l}$ represents the weight corresponding W5 to the network layer under the guidelines D.Thus, the criterion layer index and its corresponding weight and index level indicators and their corresponding final weights are given in Table 3.

\section{Fuzzy comprehensive evaluation}

The implementation of maritime emergency rescue is related to the multi-sectoral joint decision-making and coordinated implementation. Therefore, the evaluation of maritime emergency rescue capability is a complex fuzzy problem, and the fuzzy comprehensive evaluation method needs to be used to evaluate the maritime emergency rescue capability of specific maritime departments.

Fuzzy comprehensive evaluation method is based on the principle of fuzzy transformation, and according to the principle of maximum membership, the impact of multiple indicators on the system and things to make a reasonable evaluation of a method. According to the established offshore rescue capability index system, the main steps of fuzzy comprehensive evaluation of maritime emergency rescue capability are as follows:

(1) Set up index set and comment set

According to the established offshore emergency rescue capability evaluation system, the first-level indicator set $\mathrm{U}$ and the secondary index set $\mathrm{U} 1, \mathrm{U} 2, \mathrm{U} 3, \mathrm{U} 4$ :

$U=\{A, B, C, D\} \quad U 1=\{A 1, A 2, A 3, A 4\} \quad U 2=\{B 1, B 2, B 3, B 4\} \quad U 3=\{C 1, C 2, C 3, C 4\} \quad U 4=\{D 1, D 2, D 3, D 4\}$

Combined with the nature of the emergency response capability evaluation of the sea, it is divided into five qualitative grades, as follows:

$V=\{$ excellent, good, medium, qualified, bad $\}$

The index attribute excellence indicates that the index can greatly improve the maritime emergency rescue capability, and so on, the index attribute poor indicates that the index will deteriorate the maritime emergency rescue capability.

According to the established evaluation index system of emergency response capability at sea, the weights of indicators at all levels are determined by ANP, and the fuzzy evaluation model is established by combining the membership function to evaluate the emergency rescue capability of a specific emergency response unit. The synthetic algorithm of fuzzy comprehensive evaluation is shown in equation (9):

$$
B=W \circ R
$$

In the formula: $\mathrm{W}$ is the weight vector of the weight composition of the evaluation index of sea emergency rescue capability determined by ANP; R is a fuzzy relation matrix; B denotes the fuzzy comprehensive evaluation matrix of the rank; "O"is synthetic operator, $M(\bullet, \oplus): \bullet$ is defined as

$$
a \bullet b=a \times b=a b ; \oplus \text { is defined as } a \oplus b=(a+b) \wedge 1 \text { 。 }
$$

The results of the comprehensive evaluation of the reviews are the final evaluation results matrix of the fuzzy comprehensive evaluation model. According to the principle of maximum membership degree, the rating level of the element with the largest degree of membership is the final evaluation result of the fuzzy comprehensive evaluation model.

\section{The determination of the membership degree of each index}

For the evaluation index of a certain evaluation index division with membership function description, the use of isosceles triangle membership function. The mathematical description of membership of the ith index is shown in equation (10). 


$$
r_{i}\left(v_{k}\right)\left\{\begin{array}{cc}
\frac{s_{i}-m_{k}}{n_{k}-m_{k}}, & m_{k} \leq s_{i} \leq n_{k e} \\
\frac{p_{k}-s_{i}}{p_{k}-n_{k}}, & n_{k} \leq s_{i} \leq p_{k} \\
0, & \text { others }
\end{array}\right.
$$

Therefore, the fuzzy relation matrix $R_{q}$ of the q-level index is shown in equation (11).

$$
R_{q}=\left[\begin{array}{cccc}
r_{11} & r_{12} & \mathrm{~L} & r_{1 n} \\
r_{21} & r_{22} & \mathrm{~L} & r_{2 n} \\
\mathrm{M} & \mathrm{M} & \mathrm{M} \\
r_{n 1} & r_{n 2} & \mathrm{~L} & r_{n n}
\end{array}\right] \text { (11) }
$$

Comprehensive evaluation of maritime emergency rescue capability

After the membership degree of each index is determined, the comprehensive evaluation result of maritime emergency rescue capability is obtained according to the basic principle of fuzzy comprehensive evaluation, as shown in equation (12).

$$
B=W \circ R=w_{1} \circ\left(\begin{array}{l}
w_{2} \circ R_{2} \\
w_{3} \circ R_{3} \\
w_{4} \circ R_{4} \\
w_{5} \circ R_{5}
\end{array}\right)=\left(b_{1}, b_{2}, b_{3}, b_{4}, b_{5}\right)
$$

\section{Case analysis}

Taking the assessment of maritime emergency rescue capability of a sea emergency rescue department in Bohai Bay as an example, the expert scoring method is used to rate the emergency rescue capability evaluation index of the department. Fractional interval is set to 1-100. The higher the score, the better the index is. The specific score shown in Table 5:

\begin{tabular}{ccc}
\hline evaluating indicator & score \\
\hline local government A1 & 76 \\
relevant department A2 & 68 \\
Command centre A3 & 78 \\
site department A4 & 85 & 73 \\
Timely aid B1 & 83 \\
Carding site information B2 & 67 \\
Develop a rescue plan B3 & 55 \\
Configuring aid resources B4 & 96 \\
The composition of a command body C1 & 88 \\
Rapid assembly of rescue personnel C2 & 82 \\
Rapid pumping and dispatching of equipment C3 & 54 \\
Collection of traffic information C4 & 53 \\
Information management D1 & 49 \\
Resource guarantee D2 & 80 \\
Aid decision making D3 & 57 \\
Decision commandD4 & 54 \\
\hline
\end{tabular}

Table5 Indicators scoring about the emergency rescue abilities assessment

Grading the indicators at all levels and using min-max method for normalization, A standardized standard evaluation matrix is obtained, as shown in equation (13). 


$$
C_{i j}^{\prime}=\frac{C_{i j}-C_{i \max }}{C_{i \max }-C_{i \min }}
$$

In the formula: $C_{i j}$ represents the jth index in i level, and $C_{i \min }, C_{i \max }$ represent the lowest score and the highest score in i level.

Using the network analysis method to obtain the weight and weight of the index, and then use the formula (12) for fuzzy calculation:

$$
\mathrm{B}=\left[\begin{array}{lllll}
0.1075 & 0.1466 & 0.1508 & 0.1193 & 0.0836
\end{array}\right]
$$

It can be seen that the rescue capability of the emergency rescue unit at sea is qualified. The emergency rescue unit at sea can take appropriate rescue action within a certain period of time, and it meets the requirements of general emergency rescue. If further optimization is needed, the corresponding indexes can be gradually analyzed according to the index score and the index importance of ANP to improve targeted. From Table 3 and Table 5 we can see that the relevant departments A2, resource protection D2 and decision command D4 indicators of poor property, and the index weight is larger. Therefore, the department needs to strengthen the links with the relevant departments, increase the rescue resource reserves, and optimize the command and decision-making methods to improve its maritime emergency rescue capability effectively.

\section{Conclusion}

In this paper, taking relationship between the evaluation index of the sea rescue capability into account, the network analysis method is used to determine the weight of each index instead of the traditional analytic hierarchy process. Combined with the triangular membership function, the fuzzy comprehensive evaluation method is used to determine the evaluation result to evaluate the reliability of the results. The rescue department can understand its own defects through this method, and can improve the relevant indexes according to the attribute of the index and the weight determined, so as to effectively improve the rescue ability at sea. Finally, in order to verify the practicality of the evaluation model of maritime rescue capability proposed in this paper, a sea emergency rescue department in Bohai Bay is taken as an example to evaluate its maritime rescue capability. The results of case analysis show that the model can effectively evaluate the superiority of maritime rescue capability, has good practicability, and can effectively guide the relevant departments to improve. The model of the project is useful for other similar ability evaluation problems.

\section{Reference}

[1] Yang Li, Wang Lei. Assessment Index System of Coal Mine Emergency Rescue Ability Based on Interpretative Structural Modeling Method [J]. Journal of China University of Mining \& Technology (Social Science). 2015(01)

[2] Hu Junfeng, Yang Peguo. Regional Comprehensive Evaluation Index System of Disaster Reduction Capacity Based on ISM [J]. Journal of Catastrophology. 2014(01)

[3] Tan Qinwen. Study on Capability Evaluation of Maritime Emergency Response Management system [D]. Wuhan University of Technology 2010

[4] Liu Chenchen. Methodology and Application of Coal Mine Emergency Rescue Capability Evaluation [D]. Anhui University of Science \& Technology 2013

[5] Wang Qingjuan. The Path Optimization and Evaluation of HAZMAT Road Transportation Emergency Rescue System [D]. Chongqing Jiaotong University 2012 
[6] Zhang Xufeng, Zhu dan. ISM-based Analysis of Influence Factors of Basic Distribution Network Structure [J]. Logistics technology. 2010(20)

[7] Du Wen. Research on the Rescue System of Catastrophic Emergency [D]. Henan University of Technology 2012

[8] Cao Yi. Synthetic Evaluation and Application Research on the Capability of Seismic Rescue Brigade [D]. National University of Defense Technology 2008

[9] Li Lei. Mechanical Material Selection Based on FAHP [J]. Modular Machine Tool \& Automatic Manufacturing Technique. 2015(11) 